\title{
MEK5 promotes lung adenocarcinoma
}

CrossMark
To the Editor:

Lung cancer represents the leading cause of cancer death worldwide [1]. Because of that, intense efforts are being devoted to the development of novel therapeutic strategies to fight the disease [2]. In this respect, identification of new oncogenic drivers offers therapeutic opportunities in tumours in which those molecules or other cooperating elements play a pathophysiological role. Here, we show that the MEK5 mitogen-activated protein kinase kinase has a pivotal role in lung cancer.

Originally, this study was initiated with the purpose of evaluating the potential oncogenic role of the MEK5 pathway. In fact, while the MEK5 pathway has been found to be deregulated in several neoplasias [3-6], whether exclusive activation of that pathway promotes tumorigenesis has not previously been addressed. To explore that possibility, we generated transgenic mice engineered to express a constitutively active form of MEK5 by site-directed mutagenesis of the MEK5 $\mathrm{Ser}^{311}$ and $\mathrm{Thr}^{315}$ residues to aspartic acid (MEK5DD) (figure 1a). These acidic amino acid changes result in a MEK5 form in which the aspartic acid substitutions function as phosphomimetic residues [7, 8]. As a consequence, MEK5DD acts as a constitutively active kinase that is able to phosphorylate its downstream target, the ERK5 mitogen-activated protein kinase. Phosphorylation of ERK5 by constitutively active MEK5DD results in sustained activation of ERK5. Such ERK5 phosphorylation (pERK5) provokes a change in its electrophoretic mobility with respect to unphosphorylated ERK5, a characteristic that can be used to differentiate ERK5 from pERK5 by Western blotting [9]. The MEK5DD cDNA was subcloned into the pCEFL mammalian expression vector, which contains an N-terminal Flag tag sequence that serves to differentiate MEK5DD from endogenous MEK5. Increasing amounts of the cDNA coding for Flag-tagged MEK5DD were transfected in HeLa cells and its expression was analysed by Western blotting with an anti-Flag antibody. As shown in figure 1b, expression of Flag-MEK5DD caused the appearance of pERK5, indicative of pathway activation.

After this validation, SmaI and XhoI restriction sites were incorporated by PCR, and Flag-MEK5DD was then inserted into the multiple cloning site of the PMSG vector, downstream of the MMTV promoter and upstream of the SV40 polyadenylation site. This promoter has been reported to drive expression of transgenes in several tissues [10]. The construct was verified by DNA sequencing and was injected into FVB (The Jackson Laboratory, Bar Harbor, ME, USA) fertilised eggs, which were then implanted in female mice by standard procedures. All animals were maintained in a pure FVB genetic background into the specific pathogen-free area, and manipulated by authorised personnel following legal and institutional guidelines. Animal experimentation was approved by the Bioethics Committee of the University of Salamanca (authorisation number 124). Transgenic offspring identification was performed by PCR of genomic DNA from tail snips with specific MEK5DD primers. From the analysed mice, two lineages were able to stably transmit the transgene to the offspring and were therefore used for colony expansion. Mice organs were obtained after sacrifice of the animals and immediately frozen in liquid nitrogen. Western blot analyses demonstrated expression of Flag-MEK5DD in the lung, spleen, kidney, brain and breast (figure 1c).

Necropsies of these mice showed macroscopic lung masses (figure 1d) in males and females from both transgenic lineages. Tumours were not observed before 10 months of age. From a total population of 71 transgenic mice, lung tumour incidence was $46.47 \%$. Most animals developed a single tumour, although up to three lung masses could be observed in the same animal. Tumours were located in the periphery of the lung lobes and their size generally ranged from 1 to $5 \mathrm{~mm}$ in diameter. Other types of tumours were observed in some animals, although with a much lower incidence (8.45\% altogether). Tumours were found in the spleen $(n=3,4.2 \%)$, breast $(n=3,4.2 \%)$, skin $(n=5,7.04 \%)$ and the suprarenal gland $(n=1,1.4 \%)$. Some animals bore more than one tumour type. The haematoxylin-eosin histopathological analysis (figure 1e)

@ERSpublications

MEK5 acts as an oncogenic driver in mice lung cancer and is pivotal for human lung adenocarcinoma http://ow.ly/M9e830mZb8N

Cite this article as: Sánchez-Fdez A, Ortiz-Ruiz MJ, Re-Louhau MF, et al. MEK5 promotes lung adenocarcinoma. Eur Respir J 2019; 53: 1801327 [https://doi.org/10.1183/13993003.01327-2018]. 
a)

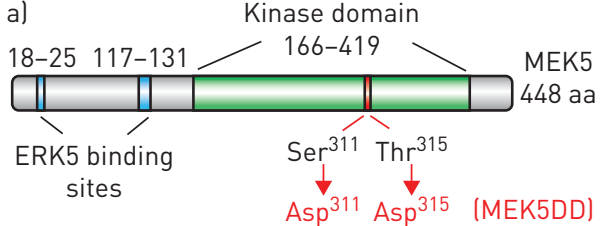

b)

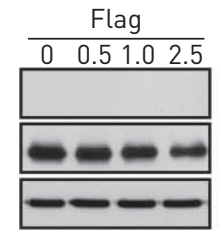

Flag-MEK5DD
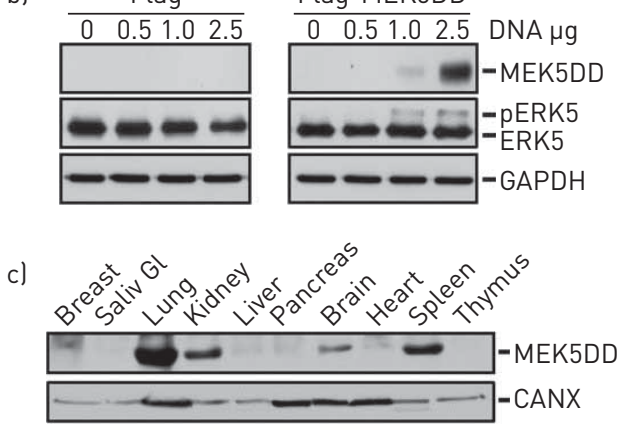

d)

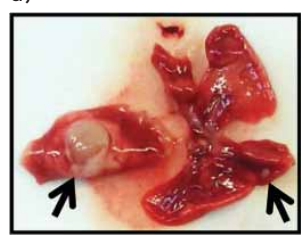

e)
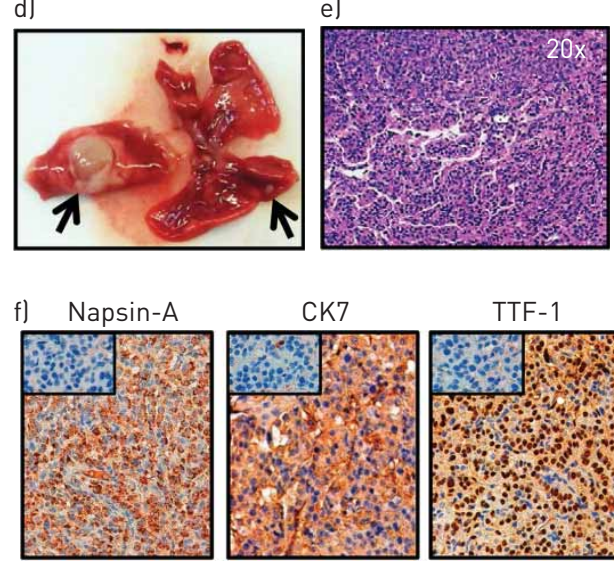

g) NT TG

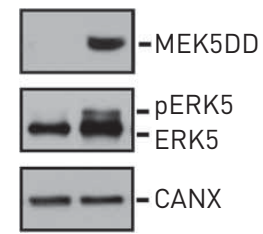

i)
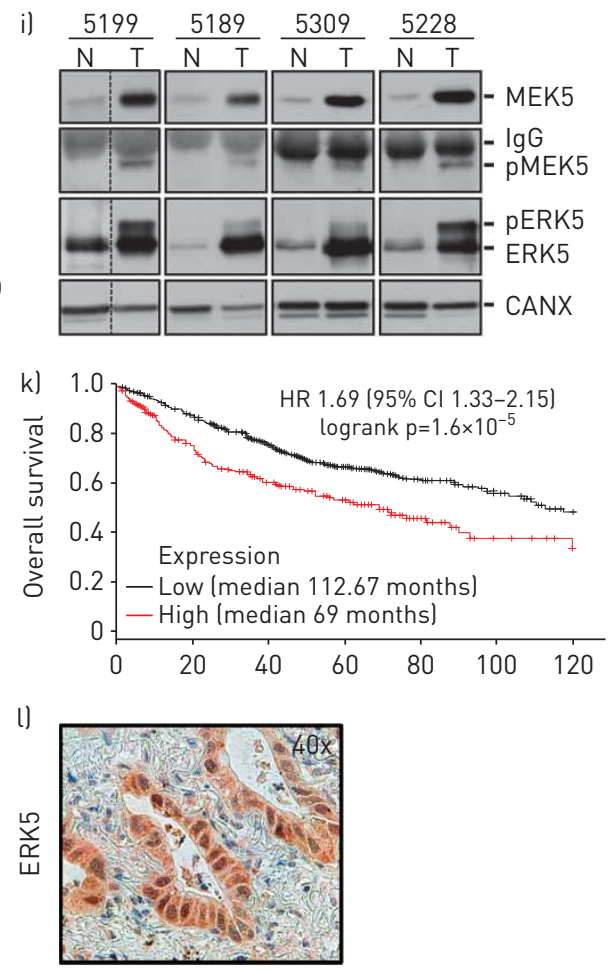
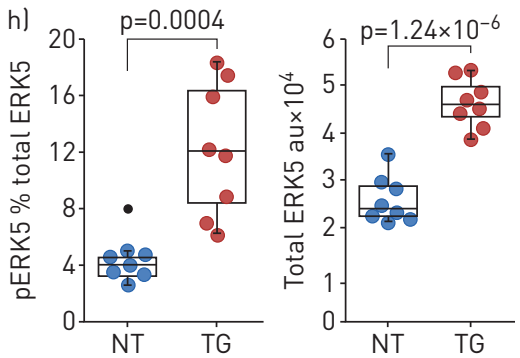

j)

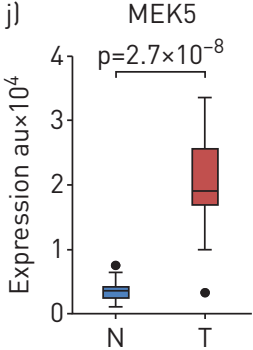

ERK5
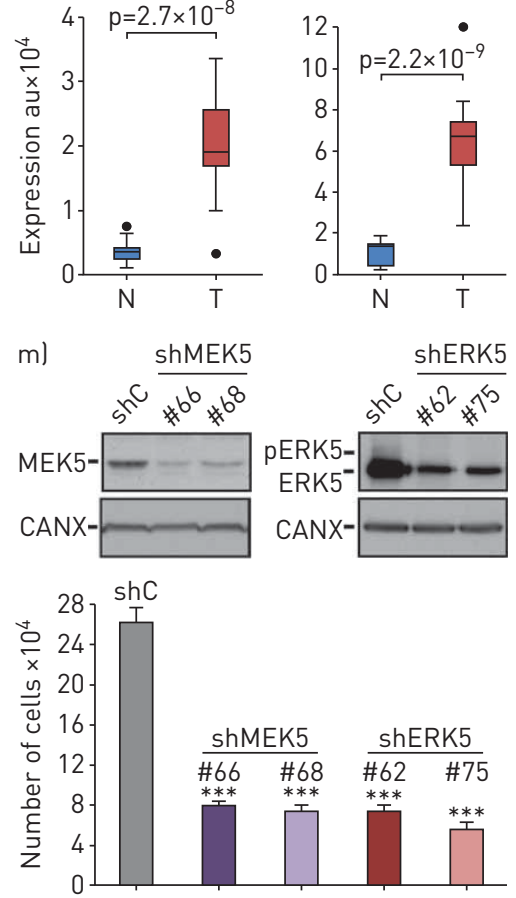

FIGURE 1 a) Schematic representation of MEK5 and the sites mutated to create constitutively active MEK5 (MEK5DD). b) The indicated amounts of pCEFL-Flag-MEK5DD were transfected into HeLa cells. ERK5 and MEK5DD were evaluated by Western blotting. c) Expression of MEK5DD in different transgenic mice tissues was evaluated by Western blotting. Note: a lane between lung and kidney was cut out from the Western blots. d) Representative macroscopic image of a MEK5DD transgenic tumoral lung (tumours are indicated by arrows). e) Representative haematoxylineosin staining of a lung mass section from a MEK5DD mouse. f) 40x representative immunohistochemical images of napsin-A, cytokeratin (CK)-7 and thyroid transcription factor (TTF)-1 of a lung adenocarcinoma from MEK5DD mouse. An inset image with an isotype control for each antibody was included. g) Western blot analysis of MEK5DD and ERK5 expression in the TG (transgenic) lung tumour compared to NT (nontransgenic) lung from a littermate mouse. h) pERK5 (left) and total ERK5 (right) levels from NT lungs ( $n=8$ ) versus TG tumoral lungs (n=8) were quantitated from Western blot analysis using ImageJ software and represented in a box plot. The median value for each group is represented as the central line of the box. Black dots represent the outlier values. Statistical comparisons were performed using SPSS 19.0 software (SPSS Inc., Chicago, IL, USA) by calculating the $p$-value according to a two-sided t-test. pERK5 levels were represented as percentage from total ERK5 expression. i) Representative Western blot analyses of MEK5, pMEK5 and ERK5 expression in human lung adenocarcinomas compared to healthy lung tissue (numbers correspond to the tissue bank classification of each patient). N: normal; T: tumour. j) Comparison between MEK5 levels (left panel) or ERK5 levels (right panel) from the total 34 human lung samples. MEK5 and ERK5 expression was quantitated as in figure $1 \mathrm{~h}$. k) 120 months follow-up KaplanMeier analyses of the relationship between combined MEK5 and ERK5 expression and overall survival in lung adenocarcinoma patients ( $\mathrm{n}=720$ ) collected in the public Kaplan-Meier plotter database. The studies were performed using the multigene classifier tool by selecting the combined mean expression values for both MEK5 (Affymetrix probe id 211370_s_at) and ERK5 (Affymetrix probe id 35617_at) genes on the 2015 version of the database. The cut-off value used to split patients into low or high expression was automatically computed by selecting the "best cut off" tool of the database. () Representative immunohistochemical analysis of the cellular location of ERK5 in human lung adenocarcinoma. $\mathrm{m}$ ) $\mathrm{NCl}-\mathrm{H} 23$ cells were infected with pLKO lentiviral vectors including short hairpin (sh) control (shC), shMEK5 or shERK5 interfering sequences. Protein expression levels were evaluated by Western blotting (top) and the proliferation (bottom) measured by cell counting. GAPDH: glyceraldehyde 3-phosphate dehydrogenase; Saliv Gl: salivary gland; CANX: calnexin; au: arbitrary units; HR: hazard ratio. ${ }^{* * *}$ : $p<0.001$.

defined the lung masses as grade I-II adenocarcinomas. Morphological inspection of the tumours showed resemblance to human lung adenocarcinomas (LUADs), since tumours were peripheral, well delimited and nonencapsulated. In fact, immunohistochemical analyses, performed using the Bond Polymer Refine Detection kit (Leica Biosystems, Newcastle, UK), presented immunoreactivity to napsin A, cytokeratin 7 and thyroid transcription factor 1, markers used to identify this subtype of tumours (figure 1f). 
Transgenic lung tumours expressed Flag-MEK5DD (figure 1g), which was accompanied by activation of ERK5. In contrast, analyses of lungs from nontransgenic littermate mice, which appeared negative for the transgene, did not show ERK5 activation. Quantitative analyses confirmed a significantly higher ERK5 activation and expression (figure $1 \mathrm{~h}$ left and right, respectively) in transgenic tumoral lungs when compared to normal lungs from nontransgenic littermates.

The above data moved us to explore the relevance of this pathway in human lung cancer. To that end, frozen human lung samples were randomly obtained from patients diagnosed with lung adenocarcinoma at the University Hospital of Salamanca, Spain. Patients provided informed consent for the use of the samples, which was approved by the Institutional Review Board Ethics Committee on Human Research of the Hospital. MEK5 and ERK5 protein activation and expression were evaluated by Western blotting in 23 LUAD samples and 11 nontumoral lung samples (nine of them corresponding to counterparts of the same LUAD patient). Constitutively active MEK5, identified using an anti-pMEK5 antibody [11], was present in tumour samples whereas no pMEK5 was detected in normal lung tissue (figure 1i). Consequently, pERK5 was observed in the LUAD samples but not in their nontumoral counterparts. Moreover, MEK5 and ERK5 expression levels were higher in tumoral samples than in normal lung tissue. Quantitative analysis evidenced a highly significant MEK5 6.2 -fold increase $\left(\mathrm{p}=2.7 \times 10^{-8}\right)$ and ERK5 5.5-fold increase $\left(p=2.2 \times 10^{-9}\right)$ in tumoral samples when compared to normal tissue (figure $1 \mathrm{j}$ ). We thus decided to explore the potential relationship between MEK5 and ERK5 expression with clinical outcome in lung cancer patients. Analysis of pooled data from the LUAD cohort of patients present in the publicly available KM-Plotter database [12] showed that high levels of combined MEK5 and ERK5 expression significantly associate with poor overall survival $\left(\mathrm{p}=1.6 \times 10^{-5}\right)$ (figure $1 \mathrm{k}$ ). Remarkably the median survival time was reduced by almost half in the patients with high MEK5/ERK5 expression (69 months) when compared to the low MEK5/ERK5 expression group (112.67 months).

Immunohistochemical analyses of human LUAD tissue revealed that ERK5 was located in the nucleus as well as in the cytoplasm of LUAD cells (figure 11). To evaluate the role of MEK5 and ERK5 in the proliferation of LUAD, RNA interference experiments were carried out on NCI-H23 cells infected with lentiviral vectors targeting MEK5 or ERK5 to decrease their expression (figure $1 \mathrm{~m}$ top). Reduction of MEK5 or ERK5 provoked a significant inhibition in the proliferation of NCI-H23 cells (figure $1 \mathrm{~m}$ bottom). Similar results were obtained using HCC4006 and NCI-H441 LUAD cell lines (data not shown), indicating that the MEK5/ERK5 axis regulates proliferation of lung adenocarcinoma cells.

Despite therapeutic improvements due to the incorporation of targeted and immune checkpoint inhibitors, lung cancer still represents the most lethal form of cancer worldwide [13]. In this respect, identification of new oncogenic drivers offers therapeutic opportunities in tumours in which those drivers play a pathophysiological role. In this article, we show that the sole activation of MEK5 fosters the appearance of lung adenocarcinomas in mice, a finding that has substantial translational interest due to its potential therapeutic implications.

The fact that the MEK5/ERK5 pathway is activated and overexpressed in LUAD samples, together with the patient clinical correlation data and its implication in the proliferation of lung adenocarcinoma cells, points to a key role of this pathway in this subtype of tumours. Moreover, it is important to notice that the major aetiopathogenic causative agent of lung cancer, i.e. tobacco smoke, provokes aberrant activation of the MEK5/ERK5 pathway [14]. In addition, certain epidermal growth factor receptor mutants found in lung cancer have been reported to activate the MEK5/ERK5 route [15].

Considering the medical need that lung cancer represents, targeting MEK5/ERK5 may offer a novel therapeutically relevant strategy in those lung adenocarcinomas in which this pathway plays a pathophysiological role. A limitation in this field is the absence of clinical stage drugs that specifically target MEK5/ERK5. Efforts to overcome this limitation could offer novel opportunities to fight this malignancy.

Adrián Sánchez-Fdez ${ }^{1,2,3}$, María Jesús Ortiz-Ruiz ${ }^{1,2}$, María Florencia Re-Louhau ${ }^{1,2}$, Isabel Ramos ${ }^{1}$, Óscar Blanco-Múñez ${ }^{2,4}$, Dolores Ludeña ${ }^{2,4}$, Mar Abad ${ }^{2,4}$, Manuel Sánchez-Martín ${ }^{3,5}$, Atanasio Pandiella ${ }^{1,2,3}$ and Azucena Esparís-Ogando ${ }^{1,2,3}$

${ }^{1}$ Instituto de Biología Molecular y Celular del Cáncer-CSIC, Salamanca, Spain. ${ }^{2}$ IBSAL, Salamanca, Spain. ${ }^{3}$ CIBERONC, Salamanca, Spain. ${ }^{4}$ Pathology Service, University Hospital, Salamanca, Spain. ${ }^{5}$ Transgenic Facility of the Nucleus Platform, University of Salamanca, Salamanca, Spain.

Correspondence: Azucena Esparís-Ogando, Instituto de Biología Molecular y Celular del Cáncer, IBSAL and CIBERONC, Campus Miguel de Unamuno, 37007-Salamanca, Spain. E-mail: esparis@usal.es

Received: July 162018 | Accepted after revision: Nov 052018 
Acknowledgements: We are grateful to M. Buschbeck (IJC, Barcelona, Spain), P. Lazo (IBMCC, Salamanca, Spain) and J. Losada (IBMCC, Salamanca, Spain) for providing us with the pCDNA3-MEK5, the pCEFL mammalian expression vector and the pMSG vector, respectively. We also thank R. Gervás Ríos and E. Alonso Morrondo (Pathology Service, University Hospital, Salamanca, Spain) for technical assistance in the immunohistochemical analyses.

Conflict of interest: None declared.

Support statement: This work was supported by grants from the Instituto de Salud Carlos III (ISCIII) (PS09/00868 and PI15/01180) to A. Esparís-Ogando. A. Sánchez-Fdez was supported by the Cancer Center Network Program from the ISCIII (RD12/0036/0003) and by the Scientific Foundation of the Spanish Association Against Cancer (AECC). Our laboratory is supported by grants from the Ministry of Economy and Competitiveness of Spain (BFU2015-71371-R to A. Pandiella), the AECC and the CRIS Cancer Foundation. Our Cancer Research Institute and the work carried out at our laboratory receive support from the European Community through the regional development funding programme (FEDER). Funding information for this article has been deposited with the Crossref Funder Registry.

\section{References}

1 Bunn PA Jr. Worldwide overview of the current status of lung cancer diagnosis and treatment. Arch Pathol Lab Med 2012; 136: 1478-1481.

2 Reck M, Hermes A, Tan EH, et al. Tissue sampling in lung cancer: a review in light of the MERIT experience Lung Cancer 2011; 74: 1-6.

3 Yang Q, Lee JD. Targeting the BMK1 MAP kinase pathway in cancer therapy. Clin Cancer Res 2011; 17: $3527-3532$.

4 Wang X, Tournier C. Regulation of cellular functions by the ERK5 signalling pathway. Cell Signal 2006; 18 : 753-760.

5 Lochhead PA, Gilley R, Cook SJ. ERK5 and its role in tumour development. Biochem Soc Trans 2012; 40: 251-256.

6 Simoes AE, Rodrigues CM, Borralho PM. The MEK5/ERK5 signalling pathway in cancer: a promising novel therapeutic target. Drug Discov Today 2016; 21: 1654-1663.

7 English JM, Pearson G, Hockenberry T, et al. Contribution of the ERK5/MEK5 pathway to Ras/Raf signaling and growth control. J Biol Chem 1999; 274: 31588-31592.

8 Nicol RL, Frey N, Pearson G, et al. Activated MEK5 induces serial assembly of sarcomeres and eccentric cardiac hypertrophy. EMBO J 2001; 20: 2757-2767.

9 Esparis-Ogando A, Diaz-Rodriguez E, Montero JC, et al. Erk5 participates in neuregulin signal transduction and is constitutively active in breast cancer cells overexpressing ErbB2. Mol Cell Biol 2002; 22: 270-285.

10 Vargo-Gogola T, Rosen JM. Modelling breast cancer: one size does not fit all. Nat Rev Cancer 2007; 7: 659-672.

11 Orive-Ramos A, Seoane S, Ocana A, et al. Regulation of the prometastatic neuregulin-MMP13 axis by SRC family kinases: therapeutic implications. Mol Oncol 2017; 11: 1788-1805.

12 Gyorffy B, Surowiak P, Budczies J, et al. Online survival analysis software to assess the prognostic value of biomarkers using transcriptomic data in non-small-cell lung cancer. PLoS One 2013; 8: e82241.

13 Brambilla E, Travis WD. Lung Cancer. In: Stewart BW, Wild CP, eds. World Cancer Report 2014. Lyon, World Health Organization (WHO), 2014; pp. 350-361.

14 Zhong CY, Zhou YM, Douglas GC, et al. MAPK/AP-1 signal pathway in tobacco smoke-induced cell proliferation and squamous metaplasia in the lungs of rats. Carcinogenesis 2005; 26: 2187-2195.

15 Jiang J, Greulich H, Janne PA, et al. Epidermal growth factor-independent transformation of Ba/F3 cells with cancer-derived epidermal growth factor receptor mutants induces gefitinib-sensitive cell cycle progression. Cancer Res 2005; 65: 8968-8974. 